\title{
A FRAMEWORK FOR THE SELECTION OF THE RIGHT NUCLEAR POWER PLANT
}

\author{
Giorgio Locatelli* \\ Politecnico di Milano \\ Dept. Management, Economics and Industrial Engineering \\ E-mail: giorgio.locatelli@polimi.it \\ Mauro Mancini \\ Politecnico di Milano \\ Dept. Management, Economics and Industrial Engineering \\ E-mail: mauro.mancini@polimi.it
}

\begin{abstract}
In the nuclear industry the vendors propose several types of Light Water Rector (LWR) with a size from 35-45 MWe up to 1600-1700 MWe. The choice of the right design is a multidimensional problem since an utility has to include not only financial factors as LCOE (Levelized Cost Of Electricity), IRR (Internal Rate of Return), but also the so called "External Factors" like the required spinning reserve or the impact on the local industry or the social acceptability. The international literature proposes several techniques to solve this multidimensional problem, unfortunately it seems not possible to apply these methodologies as they are, since the problem is too complex and it is difficult to provide in a reliable way the needed experts judgments. This paper try to fill this gap proposing a two steps framework to choose the best nuclear reactor at pre-feasibility study phase.
\end{abstract}

Keywords: Small Medium Reactors, Investment Evaluation, NPP selection, External Factors

\section{INTRODUCTION}

The "Politecnico di Milano" university is developing a model called INCAS (INtegrated model for the Competitiveness Assessment of SMRs) to assess strengths and weaknesses of Small-Medium Reactors (SMRs). SMR have electrical output lower than 700MWe, while Large Reactors (LRs) have an electrical output higher than 700 MWe (IAEA, 2006). INCAS compares the choice of investment in SMRs or LRs providing monetary and not monetary (external) indicators. (Boarin \& Ricotti, 2009) presents economic and financial comparison of Large and Small-Medium designs, while not monetary factors (from now on external factors) are addressed in (Locatelli and Mancini, 2011). An "External Factor" is defined as a factor not included in traditional Discounted Cash Flow Methods (DCFM) for the evaluation of investments, because of its qualitative and subjective nature, but which is able to

\footnotetext{
* Corresponding author
} 
heavily affect the investment attractiveness. (Locatelli and Mancini, 2011) lists and explains External Factors which are differential for the choice between LRs and SMRs. As general rules the LRs perform better considering traditional economic and financial aspects (like IRR and LUEC) while SMRs perform better or similar in the external factors (not easy to quantify). The goal of this paper is to define a framework to integrate both contributions to chose the most suitable Nuclear Power Plant (NPP).

\section{LITERATURE REVIEW}

The integration of Financial and External Factors performances requires the application of Multi Attribute Decision Making (MADM) methods, which deal with the problem of choosing the best solution among a finite set of alternatives (Ribeiro, 1996). Saaty's Analytic Hierarchy Process (AHP) (Saaty, 1980), is one of the most used methods, because of its ability to fit different problems. It could be also implemented through a fuzzy approach, which permits to elicit expert opinions using linguistic variables. Fuzzy AHP seems to follow better the human thinking (Deng, 1999) because every pairwise comparison has not attached a precise ratio number, but a fuzzy set which takes uncertainties into account (Hsieh et al., 2004). The main problem of fuzzy version is the complex and unreliable process of ranking fuzzy sets resulting from evaluation of alternatives (Leung \& Cao, 2000). Outranking methods are usually employed in the prioritization of many alternatives but some of them, like ELECTRE (Georgopoulous, 1997), or PROMETHEE (Nowack, 2005), have the advantage to be based on a global preference model, expressed by preference and indifference thresholds, which permit to express different degrees of preference between two alternatives. The main weakness is the high number of thresholds values required to the decision maker: this strongly increases the complexity of the decision process. TOPSIS approach is intuitively appealing and easy to understand (Opricovic \& Tzeng, 2004): it is based on the assumption that the best alternative should have the shortest Euclidean distance from an ideal positive solution (made up of the best value for each attribute regardless of alternative) and the farthest distance from a negative ideal solution (made up of the worst values). Respect to outranking methods, further thresholds or parameters are not required. Each performance can be considered in the model through its specific measurement.

\section{THE TWO-STEP PROCESS}

The choice of the right MADM technique requires a deep analysis of strengths and weaknesses of each method: some have a solid and reliable mathematic base, others can be implemented in a simpler way (Kiker et al., 2005). Table 1 provides a short summary. In the selection process of choosing the best size of a Nuclear Power Plant different solutions are evaluated on the base of Financial and External attributes that must be suddenly weighted and finally combined through MADM techniques for the final prioritization. So, it is useful to separate MADM methods in two different groups: 
1. Methods requiring importance weights as inputs from external sources: they are Scoring Methods, TOPSIS, ELECTRE and PROMETHEE. These require to use other techniques to get weights.

2. Methods which calculate importance weights as part of their integration process, (AHP and its fuzzy version). So, AHP and fuzzy AHP could be implemented in two different ways: to support the whole process, till final prioritization or to determine only importance weights

\begin{tabular}{|c|c|c|c|}
\hline & METHOD & & STRENGTHS and WEAKNESSES \\
\hline \multirow{4}{*}{ 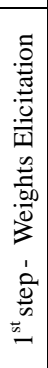 } & \multirow[b]{2}{*}{ AHP } & + & There are dedicated software which simplify elicitation from experts and final ranking \\
\hline & & - & $\begin{array}{l}\text { It does not take into account the uncertainty associated with the mapping of human judgment to a number (Yang \& } \\
\text { Chen, 2004). Experts must give crisp numerical judgments of relative importance of each attribute on each other } \\
\text { Experts must judge "how many times" an attribute is more important than another }\end{array}$ \\
\hline & \multirow{2}{*}{$\begin{array}{l}\text { Fuzzy } \\
\text { AHP }\end{array}$} & + & $\begin{array}{l}\text { Experts have not to express "how many times" an attribute is more important. They express their opinion through } \\
\text { simple linguistic judgments: questionnaire is easier to understand, faster to be filled and so resulting weights are more } \\
\text { accurate } \\
\text { Overlapping of fuzzy judgments well considers uncertainty and vagueness of the subjective perception }\end{array}$ \\
\hline & & - & $\begin{array}{l}\text { Mathematic elaboration is more complicated, but only if method is used for the final integration }\left(2^{\text {nd }} \text { step }\right) \\
\text { No dedicated software } \\
\text { Less experienced method, both in theory and real case applications. }\end{array}$ \\
\hline \multirow{8}{*}{ 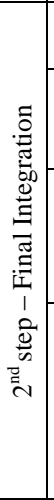 } & \multirow{2}{*}{$\begin{array}{l}\text { Scoring } \\
\text { Meth. }\end{array}$} & + & Simple and easy to be understood \\
\hline & & - & It is difficult to find a unique function able to represent the relationships among performances \\
\hline & \multirow[b]{2}{*}{ ELECTRE } & + & Decision makers can customize the process fixing different thresholds for the indexes \\
\hline & & & $\begin{array}{l}\text { Thresholds strongly affect the final ranking and make it subjective, requiring too information from decision maker } \\
\text { More useful with many alternatives and few attributes }\end{array}$ \\
\hline & \multirow[b]{2}{*}{$\begin{array}{l}\text { PROM } \\
\text { ETHEE }\end{array}$} & + & Decision makers can customize the process fixing different thresholds for the indexes \\
\hline & & - & $\begin{array}{l}\text { It requires the elicitation of a preference and an indifference threshold value for each attribute. Process is more } \\
\text { complicated and the higher request of information does not guarantee a better ranking of designs, considering that } \\
\text { decision maker is dealing with ballpark estimates in selection phase. }\end{array}$ \\
\hline & \multirow[t]{2}{*}{ TOPSIS } & + & $\begin{array}{l}\text { Simple and easy to be understood } \\
\text { It considers the effective difference between values on each attribute for different NPP designs } \\
\text { Every performance can be evaluated using its specific unit of measurement } \\
\text { It does not require more information, threshold values or parameters from decision maker. The process is simpler and } \\
\text { less subjective. }\end{array}$ \\
\hline & & - & More useful with many alternatives \\
\hline
\end{tabular}

Table 1 - Critical review of MADM methods for "Selection of the best NPP design. (+) Advantages, (-) Disadvantages

Finally, the choice is between a one-step and a two-step MADM process. In the first, AHP or fuzzy AHP use experts elicitation based on pairwise comparisons, along the hierarchical structure form of the problem, to get prioritization and final ranking of the projects. In the second, AHP or fuzzy AHP can be used to get importance weights through expert, stakeholders and decision makers elicitation; then weights will be integrated with financial and external performances of NPP designs, using Scoring Methods, TOPSIS, ELECTRE or PROMETHEE. AHP and fuzzy AHP are the unique techniques able to get importance weights among the methods considered in this paper. Scoring methods, ELECTRE, PROMETHEE and TOPSIS are available for the final integration ( $2^{\text {nd }}$ phase). According to Table 1, we suggest the choice of fuzzy AHP and TOPSIS methods. Fuzzy version of AHP takes into consideration the uncertainty on judgements from experts and, above all, it avoids the need to express pairwise judgement in form of crisp numerical value, as for traditional AHP. Fuzzy AHP is excellent to get weights from experts elicitation, as demonstrated by numerous similar applications in literature e.g. (Kahraman et al., 2004), (Kahraman \& Cebi, 2009). TOPSIS will be exploited for the final integration because it is really simple and easy to be understood: these are the most important characteristics for a tool supporting selection and pre-feasibility phases. Many 
parameters required by other methods would make the $2^{\text {nd }}$ step too complicated, without ensuring a more precise evaluation because in the selection phase decision makers are still dealing with ballpark estimates. On the base of all previous consideration, the complete process for selection of the best NPP size for a certain scenario can be summarized in 6 points:

1. Identification of relevant attributes for evaluation and selection, looking at the specific country taken into consideration.

2. Definition of measurement and evaluation process of each attribute: quantitative or qualitative, monetary or not, etc... Each NPP design will have to be evaluated on each attribute.

3. Definition of attribute's hierarchical structure as required for fuzzy AHP application.

4. Experts elicitation to get attributes' weights. Each expert has to fill in a questionnaire of pairwise comparisons between attributes or group of them. Fuzzy AHP permits to express judgments through linguistic variables: each one is linked to a triangular fuzzy number following the scale in (Yang \& Chen, 2004).

5. Pairwise comparisons matrices from different decision makers are aggregated through the geometric mean method presented in (Kuo et al., 2002). Buckley's method (Buckley, 1985), is then applied to up the hierarchical structure and to get final importance weights. These are fuzzy sets, so a defuzzification process is needed to obtain crisp values: the most common is the Centroid Method (Opricovic \& Tzeng, 2004).

6. TOPSIS is applied for the final integration, looking at the 5 steps in (Hwang \& Yoon, 1981) and (Opricovic \& Tzeng, 2004).

\section{SELECTING THE BEST NPP IN A GIVEN SCENARIO}

The 6-points complete method was applied to define which NPP size, between LR and SMR, fits well characteristics and needs of the Italian scenario.

Point 1-2. INCAS evaluated the overall performances respect to each attribute. First two points are carried out in the development of INCAS: decision makers, experts and literature review indicated 17 relevant attributes to evaluate NPP projects' attractiveness.

Point 3. The hierarchical structure for the implementation of fuzzy AHP is presented in Figure 1

Point 4. Importance weights of INCAS' attributes are strictly country-dependent, so their elicitation from experts is really the best way to get them. Elicitation is obtained through a questionnaire designed for fuzzy AHP, following the scheme in (Ozdagoglu \& Ozdagoglu, 2007). The questionnaire was composed by 34 questions and 22 experts (out of 40) filled it.

Point 5-6. The defuzzified weights obtained from the application of geometric mean and Buckley's 
methods are summarized in a table 2. It also shows the best performing solution on each attribute and final indexes (relative Euclidean closeness to ideal solution) for LRs and SMRs. The main goal of the table is to highlight which attributes promote LR choice in the Italian scenario, and which ones promote SMRs. The two-step process shows the best NPP in the Italian scenario is the LR

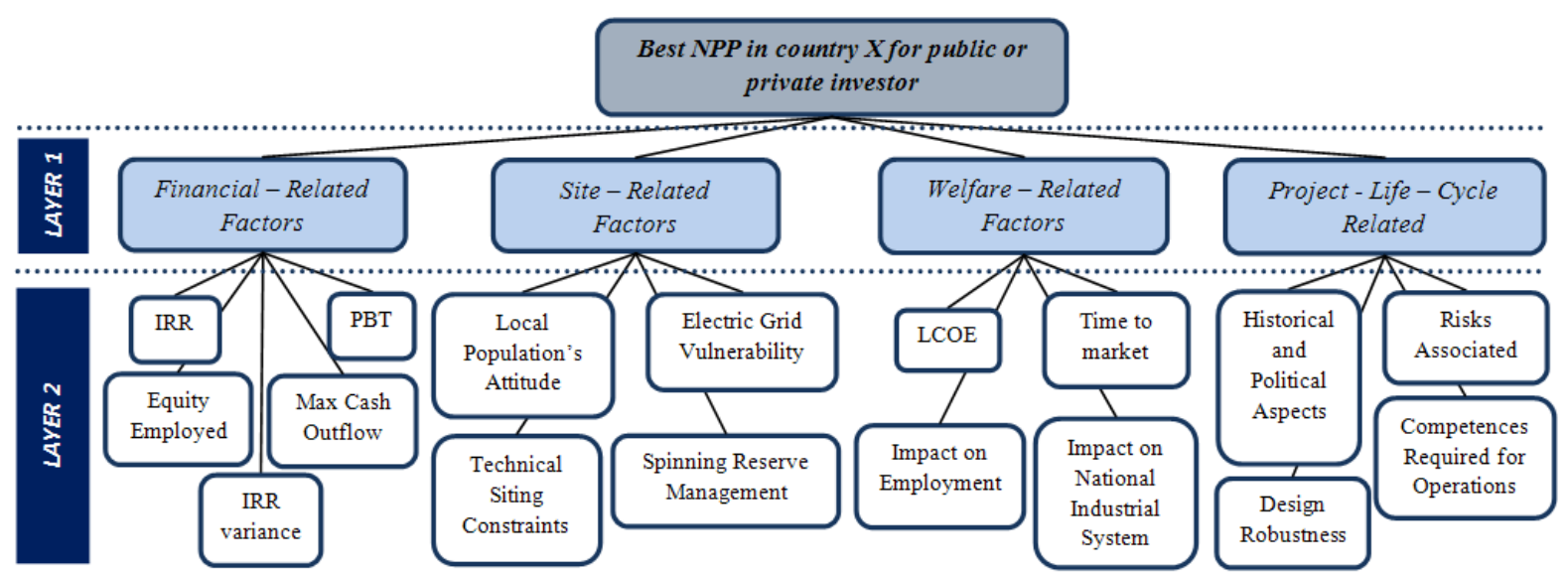

Figure 1 - Hierarchical structure for weights' elicitation using fuzzy AHP

\begin{tabular}{|c|c|c|c|c|c|}
\hline ATTRIBUTE & \begin{tabular}{|l|}
$\begin{array}{l}\text { Class } \\
\text { factors }\end{array}$ \\
\end{tabular} & Weights of class & $\begin{array}{l}\text { Weights of attributes } \\
\text { in the class }\end{array}$ & $\begin{array}{l}\text { Absolute weights } \\
\text { of attributes }\end{array}$ & Best performance \\
\hline IRR & \multirow{5}{*}{$\begin{array}{c}\text { Financial } \\
\text { related }\end{array}$} & \multirow{5}{*}{$30,1 \%$} & \begin{tabular}{|c|}
$29,7 \%$ \\
\end{tabular} & $8,9 \%$ & LR \\
\hline IRR variance & & & $20,7 \%$ & $6,2 \%$ & Roughly equal \\
\hline Payback Time & & & $20,1 \%$ & $6,0 \%$ & Roughly equal \\
\hline Equity employed & & & $17,2 \%$ & $5,2 \%$ & LR \\
\hline Max cash outflow & & & $12,4 \%$ & $3,7 \%$ & SMR \\
\hline Spinning reserve & \multirow{4}{*}{$\begin{array}{c}\text { Site } \\
\text { related }\end{array}$} & \multirow{4}{*}{$24,9 \%$} & $7,4 \%$ & $1,8 \%$ & SMR \\
\hline Grid vulnerability & & & $13,2 \%$ & $3,3 \%$ & SMR \\
\hline Local population's attitude & & & $56,5 \%$ & $14,1 \%$ & Roughly equal \\
\hline Technical siting constraints & & & $23,0 \%$ & $5,7 \%$ & SMR \\
\hline Time to market & \multirow{5}{*}{$\begin{array}{l}\text { Welfare } \\
\text { related }\end{array}$} & \multirow{5}{*}{$24,0 \%$} & $15,0 \%$ & $3,6 \%$ & SMR \\
\hline Impact on employment (construction) & & & $5,8 \%$ & $1,4 \%$ & SMR \\
\hline Impact on employment (operation) & & & $5,8 \%$ & $1,4 \%$ & SMR \\
\hline Impact on national industrial system & & & $20,5 \%$ & $4,9 \%$ & SMR \\
\hline Levelized Cost Of Electricity & & & $53,0 \%$ & $12,7 \%$ & LR \\
\hline Risk associated to the project & \multirow{4}{*}{$\begin{array}{c}\text { Project } \\
\text { Life Cycle } \\
\text { related }\end{array}$} & \multirow{4}{*}{$21,0 \%$} & $33,0 \%$ & $6,9 \%$ & SMR \\
\hline Design Robustness & & & $22,1 \%$ & $4,6 \%$ & SMR \\
\hline Historical and political aspect & & & $32,2 \%$ & $6,7 \%$ & LR \\
\hline Competences required for operations & & & \begin{tabular}{l|l}
$12,8 \%$ & \\
\end{tabular} & $2,7 \%$ & \begin{tabular}{|c|} 
SMR \\
\end{tabular} \\
\hline \begin{tabular}{|l|} 
Final Index $C_{S M R}$ \\
Final Index $C_{L R}$
\end{tabular} & & $\begin{array}{l}0,4623 \\
0,5377\end{array}$ & \multicolumn{3}{|c|}{$\begin{array}{c}\text { The best solution has the highest value of C: LRs are slightly better in } \\
\text { the Italian case }\end{array}$} \\
\hline
\end{tabular}

Table 2 - Weights and final integration results for best NPP technology in the Italian scenario

\section{CONCLUSIONS}

Traditional Discounted Cash Flow methods for the evaluation of investments are not able to consider a complete set of factors (External Factors) because of their qualitative and subjective nature, but they can heavily affect the attractiveness of different designs of NPP. The two-steps process is a valuable tool to support the decision making process in selecting the right nuclear power plant for a certain country: in the first phase, fuzzy AHP will be used to obtain the importance weights of factors: it permits to consider experts opinions in the simplest and most efficient way; resulting weights will be used for the integration of LRs' and SMR' performances, on Financial and External Factors, through 
TOPSIS method, a simple and understandable MADM technique. The final outcome is a unique, numerical and crisp index, which permits to rank alternatives. This work includes an application of the new framework to the Italian scenario, even if most of the considerations can be applied to many countries.

\section{REFERENCES}

Boarin, S. \& Ricotti, M.E., (2009). Cost and profitability analysis of modular SMRs in different deployment scenarios, Paper 75741. ICONE 17 July 12-16. Brussels, Belgium, 2009.

Buckley, J.J., (1985). Rankinhg alternatives using fuzzy numbers. Fuzzy Sets and Systems, 15, 1-31.

Deng, H., (1999). Mulricriteria Analysis with Fuzzy Pairwise Comparison. IEEE International Fuzzy Systems Conference Proceedings. August, 22-25. Seoul, Korea.

Georgopoulous, E., (1997). A Multi-Criteria Decision Aid approach for energy planning problems: the case of renewable energy option. European Journal of Operational Research, 103, 38-54.

Hsieh, T.Y., Lu, S.T. \& Tzeng, G.H., (2004). Fuzzy MCDM approach for planning and design tenders selection in public office buildings. International Journal of Project Management, 22, 573-84.

Hwang, C.L. \& Yoon, K.L., (1981). Multiple Attribute Decision Making: Methods and Applications. New York: SpringerVerlag.

IAEA, (2006). Status of innovative small and medium reactor designs 2006. Reactors with conventional refueling schemes, IAEA-TECDOC-1485. Vienna, Austria: IAEA.

Kahraman, C. \& Cebi, S., (2009). A new multi-attribute decision making method: Hierarchical fuzzy axiomatic design. Expert Systems with Applications, 36, 4848-61.

Kahraman, C., Cebeci, U. \& Ruan, D., (2004). Multi-attributi comparison of catering service companies using fuzzy AHP: The case of Turkey. International Journal of Production Economics, 87, 171-84.

Kiker, G.A. et al., (2005). Application of Multicriteria Decision Analysis in Environmental Decision Making. Integrated Environmental Assessment and Management, 1(2), 95-108.

Kuo, R.J., Chi, S.C. \& Kao, S.S., (2002). "A decision support system for selecting convenience store location through integration of fuzzy AHP and artificial neural network". Computers in Industry, 47, 199-214.

Leung, L.C. \& Cao, D., (2000). On consistency and ranking of alternatives in fuzzy AHP. European Journal of Operational Research, 124, 102-13.

Locatelli G., Mancini M. (2011); The role of the reactor size for an investment in the nuclear sector: an evaluation of notfinancial parameters. Progress in Nuclear Energy. 53 (2), 212-222

Nowack, M., (2005). Investment projects evaluation by simulation and multiple criteria decision aiding procedure. Journal of Civil Engineering and Management, 11(3), 193-202.

Opricovic, S. \& Tzeng, G.H., (2004). Compromise solution by MCDM methods: A comparative analysis of VIKOR and TOPSIS. Europen Journal of Operational Research, 156, 445-55.

Ozdagoglu, A. \& Ozdagoglu, G., (2007). Comparison of AHP and Fuzzy AHP for the Multi-Criteria Decision Making Process with linguistic evaluations. Instanbul Ticaret Universitesi Fen Bilimleri Yil, 65-85.

Ribeiro, R.A., (1996). Fuzzy multiple attribute decision making: a review and new preference elicitation techniques. Fuzzy Sets and Systems, 78(2), 155-81.

Saaty, T.L., (1980). The Analytic Hierarchy Process. Mc Graw-Hill. New York, NY, USA. 